\title{
Isolated septal myectomy for hypertrophic obstructive cardiomyopathy: an update on the Toronto General Hospital experience
}

\author{
Rachel D. Vanderlaan ${ }^{1}$, Anna Woo ${ }^{2}$, Anthony Ralph-Edwards ${ }^{1,3}$ \\ ${ }^{1}$ Division of Cardiac Surgery, the University of Toronto, Toronto, Canada; ${ }^{2}$ Division of Cardiology, ${ }^{3}$ Division of Cardiac Surgery, University Health \\ Network, Toronto, Canada \\ Correspondence to: Anthony Ralph-Edwards. Division of Cardiovascular Surgery, University Health Network, 4N-448 Toronto General Hospital, 200 \\ Elizabeth Street, Toronto, ON, M5G 2C4, Canada. Email: Anthony.ralph-edwards@uhn.ca.
}

\begin{abstract}
Background: Isolated septal myectomy is considered the gold standard for refractory left ventricular outflow tract (LVOT) obstruction at centers with dedicated hypertrophic obstructive cardiomyopathy (HOCM) surgeons. In this paper, we provide an update on the Toronto General Hospital (TGH) experience for isolated septal myectomy and comment on the safety and efficacy of myectomy in patients with thin basal septal thickness at our institution.
\end{abstract}

Methods: We retrospectively reviewed all patients undergoing surgical myectomy at our institution from January 2012 to August 2016. We analyzed patient characteristics, intraoperative variables, pre- and postprocedural echocardiographic parameters, and key outcomes including post-operative stroke, renal failure, iatrogenic ventricular septal defect (VSD), post-procedure insertion of permanent pacemaker, and mortality. Results: At our institution, 150 isolated septal myectomy surgeries were performed over the study period. Preoperative echocardiography demonstrated an average basal septal thickness of $2.10 \pm 0.43 \mathrm{~cm}$ with a resting gradient of $67 \pm 37 \mathrm{mmHg}$ and a provoked gradient of $89 \pm 40 \mathrm{mmHg}$. Fifty percent of patients had significant systolic anterior motion (SAM) of their mitral valve and $53 \%$ had associated moderate to severe mitral regurgitation (MR). Of note, $24 \%$ of patients had a thin septum of $\leq 1.7 \mathrm{~cm}$. Discharge echocardiograms demonstrated significant septal reduction to an average basal septal thickness of $1.04 \pm 0.26(\mathrm{P}<0.05)$, with negligible resting and provokable LVOT gradients. At the time of discharge, none of the patients had significant SAM and only $5.3 \%$ of patients had residual greater than mild MR. Patients undergoing isolated myectomy with a thin basal septum had similar outcomes to those with a $>1.7 \mathrm{~cm}$ septal thickness. In our contemporary cohort, there were no iatrogenic VSDs, $5.3 \%$ of patients required a permanent pacemaker and there was one early death.

Conclusions: Our cohort demonstrates that isolated septal myectomy remains a safe and effective operation that can achieve excellent results, irrespective of basal septal thickness, when done by experienced surgeons in a dedicated hypertrophic cardiomyopathy (HCM) center.

Keywords: Hypertrophic obstructive cardiomyopathy (HOCM); septal myectomy; systolic anterior motion of mitral valve

Submitted Mar 03, 2017. Accepted for publication Apr 20, 2017.

doi: $10.21037 /$ acs.2017.05.12

View this article at: http://dx.doi.org/10.21037/acs.2017.05.12 


\section{Introduction}

Hypertrophic obstructive cardiomyopathy (HOCM) is characterized by left ventricular outflow tract (LVOT) obstruction due to asymmetric septal hypertrophy and systolic anterior motion (SAM) of the mitral valve. Patients who fail medical management and have ongoing symptoms of dyspnea, chest pain, or syncope can be managed by alcohol septal ablation (ASA) or surgical myectomy $(1,2)$. In addition, some centres offer concomitant mitral valve surgery for primary treatment of LVOT obstruction and, most recently, the mitral clip procedure has been used for high-risk surgical patients $(3,4)$.

Surgical resection of muscle from the subaortic region to enlarge the LVOT results in relief of SAM of the mitral valve and its resultant mitral regurgitation (MR), and is the gold standard for refractory LVOT obstruction at centers with dedicated HOCM surgeons $(1,5)$. In dedicated HOCM centres, mortality is typically less than $1 \%$ for isolated myectomies and is associated with excellent long-term survival (1,6-8).

Our institution has a dedicated hypertrophic cardiomyopathy (HCM) program and both of the major septal reduction strategies, septal myectomy and ASA, are offered. Our centre has generally performed ASA in patients with a suitable septal perforator branch and appropriate septal morphology (focal basal septal hypertrophy and color turbulence in the outflow tract). When comparing the two procedures at our centre, ASA is associated with a higher incidence of permanent pacing (9). In agreement with this data, larger series have demonstrated that patients greater than 65 years of age have greater survival, freedom from severe symptoms, and improved quality of life with isolated myectomy compared with ASA $(10,11)$.

A distinct group within symptomatic HOCM patients are those patients with thin basal septal thickness. Many centers offer ASA for symptomatic mild asymmetric hypertrophy and septal myectomy for those with significant hypertrophy, due to concerns of iatrogenic ventricular septal defects (VSDs), as well as the morbidity and mortality associated with advanced age in patients with mild septal hypertrophy. In addition, it is thought that the mechanism of LVOT obstruction in the absence of significant asymmetric hypertrophy may have contributions from abnormalities in the mitral-subvalvular apparatus, and some centres elect to perform concomitant mitral procedures to target this distinct population (3). In this paper, we provide an update on the TGH experience for isolated septal myectomy and comment on its safety and efficacy in patients with thin basal septal thickness at our institution.

\section{Methods}

We retrospectively reviewed all patients undergoing myectomy surgical procedure [Morrow type resection (7)] at our institution from January 2012 to August 2016. All patients with isolated septal myectomy (including those undergoing concomitant LAD muscle bridge division, atrial fibrillation, and aortoplasty procedures) were analyzed for patient characteristics, intraoperative variables, and pre- and post-procedural echocardiographic parameters. Descriptive statistics such as proportion mean with standard deviation and median with interquartile range (IQR) were used as appropriate. Statistical comparisons were done by $t$-tests and chi square or Fisher exact tests when appropriate. A $\mathrm{P}$ value of less than 0.05 indicated statistical significance. Key outcomes included post-operative stroke, renal failure, iatrogenic VSD, post procedure insertion of permanent pacemaker, and mortality.

\section{Results}

\section{Major cardiac surgical procedures for HOCM patients}

At our institution, between January 2012 and August 2016, 150 isolated septal myectomy surgeries were performed using a Morrow type resection (7). In addition, there were 65 patients who underwent myectomy with concomitant coronary artery bypass grafting (CABG), 17 patients who underwent myectomy with mitral valve repair or replacements, and 59 patients who underwent myectomy and other concomitant cardiac surgery, such as combined valve and CABG procedures (Table 1). Of those patients undergoing isolated septal myectomy, $6 \%(n=9)$ had a concomitant atrial fibrillation procedure.

\section{Patient characteristics of HOCM patients undergoing isolated septal myectomy}

In the cohort of patients undergoing isolated septal myectomy, the median age at time of operation was 52 years with $62 \%$ of the patients being male. The majority of patients had severe symptoms refractory to medical management, with $65 \%$ of patients reporting NYHA class III-IV symptoms, and 58\% reporting CCS class III-IV symptoms at the time of surgery. In addition, $27 \%$ reported 


\begin{tabular}{ll} 
Table 1 Major cardiac surgical procedures for HOCM patients \\
\hline Cardiac surgical procedures & Cohort $(n=291)$ \\
\hline Isolated septal myectomy & 150 \\
\hline Myectomy + CABG & 65 \\
\hline Myectomy + mitral repair/replacement & 17 \\
\hline Myectomy + other & 59 \\
\hline
\end{tabular}

HOCM, hypertrophic obstructive cardiomyopathy; CABG, coronary artery bypass grafting.

having syncope prior to surgical intervention (Table 1).

\section{Surgical relief of LVOT obstruction}

Preoperative characteristics on echocardiography demonstrated an average basal septal thickness of $2.10 \pm 0.43 \mathrm{~cm}$, with a resting gradient of $67 \pm 37 \mathrm{mmHg}$ and a provoked gradient of $89 \pm 40 \mathrm{mmHg}$. Fifty percent $(\mathrm{n}=75)$ of patients had significant SAM on their preoperative echocardiogram and 53\% ( $\mathrm{n}=79)$ had associated moderate to severe MR. The average left atrial size was $4.5 \pm 0.7 \mathrm{~cm}$ and the right ventricular systolic pressure (RVSP) was $33 \pm 10 \mathrm{mmHg}$ in this cohort.

The average cardiopulmonary bypass time was $58 \pm 13$ minutes with a cross clamp time of $38 \pm 11$ minutes. Postoperatively, patients were extubated after 4 (IQR, 2.65.8 ) hours, the length of stay in intensive care was a median of 26 (IQR, 24-48) hours, and time to discharge was 6 (IQR, 5-7) days.

Discharge echocardiograms demonstrated significant septal reduction to an average basal septal thickness of $1.04 \pm 0.26(\mathrm{P}<0.05)$. Resting LVOT gradients were $11 \pm 7 \mathrm{mmHg}$, with no significant increase in provokable gradient $(13 \pm 9 \mathrm{mmHg})$. At the time of discharge, none of the patients had significant SAM and only $5.3 \%$ of patients had residual greater than mild MR (Table 2). Of those with residual MR at time of discharge, available follow-up echocardiograms revealed only mild MR.

Of note, $24 \%(n=36)$ of patients had mild asymmetric hypertrophy, with a resting basal septum of $\leq 1.7 \mathrm{~cm}$. In this contemporary cohort, patients with a basal septal thickness $\leq 1.7 \mathrm{~cm}$ were 57 (range 48-62) years of age, compared to 51 (range 40-59) years of age with a septum greater than $1.7 \mathrm{~cm}$. Resting $(67 \pm 37$ vs. $67 \pm 40 \mathrm{mmHg})$ and provokable gradients $(88 \pm 39$ vs. $94 \pm 43 \mathrm{mmHg})$ and the amount of patients with significant SAM or significant MR were not

\begin{tabular}{ll}
$\begin{array}{l}\text { Table } 2 \text { Preoperative characteristics for isolated septal myectomy } \\
\text { cohort }\end{array}$ & Value (cohort $n=150)$ \\
\hline Patient preoperative variable & $52[42-61]$ \\
\hline Median age (years) & $93(62 \%)$ \\
\hline Sex (male) & $55[40-63]$ \\
\hline Median age for females (years) & $51[44-58]$ \\
\hline Median age for males (years) & $2.04(1.85-2.2)$ \\
\hline BSA & $98(65 \%)$ \\
\hline NYHA class (III-IV) & $87(58 \%)$ \\
\hline CCS class (III-IV) & $40(27 \%)$ \\
\hline Syncope & $36(24 \%)$ \\
\hline Basal septal thickness $\leq 1.7$ cm & $9(6 \%)$ \\
\hline Concomitant atrial fibrillation procedure & 9 Herk Heart Association; \\
\hline $\begin{array}{l}\text { BSA, body surface area; NYHA, New } \\
\text { CCS, Canadian Cardiovascular Society. }\end{array}$ \\
\hline
\end{tabular}

different between those with a basal septal thickness $\leq 1.7 \mathrm{~cm}$ versus those with a septum thickness greater than $1.7 \mathrm{~cm}$. Post-operatively, both groups had negligible gradients, with no residual SAM or significant MR on their discharge echocardiogram (Table 3). Overall, there were no deaths or iatrogenic VSDs in the thin septal thickness group.

\section{Outcomes for isolated myectomy patients}

Within this contemporary cohort, there was one death due to heart failure attributed to severe diastolic dysfunction and post-operative renal failure. In our cohort, three patients $(2 \%)$ had a diagnosis of a postoperative cerebrovascular event, with two having mild neurological deficits. In the 150 patients undergoing isolated septal myectomy, there were no iatrogenic VSDs and a permanent pacemaker was required in $5.3 \%(n=8)$ of patients, of whom four had a preoperative electrocardiogram with a right bundle branch block. In addition, three patients had an automated implantable cardioverter defibrillator (AICD) placed post-operatively.

\section{Discussion}

In agreement with results from dedicated HCM centres, surgical relief of LVOT obstruction by surgical myectomy is both safe and efficacious. In this contemporary cohort spanning approximately four years, early postoperative 


\begin{tabular}{lll}
\hline Table 3 Echocardiography parameters & & Discharge Echo \\
\hline Parameter & Preoperative Echo & $1.04 \pm 0.26^{*}$ \\
\hline Basal septal thickness $(\mathrm{cm})$ & $2.10 \pm 0.43$ & $11 \pm 7^{*}$ \\
\hline Resting LVOT gradient $(\mathrm{mmHg})$ & $67 \pm 38$ & $13 \pm 9^{*}$ \\
\hline Provoked LVOT gradient $(\mathrm{mmHg})$ & $89 \pm 40$ & $0(0 \%)^{\star}$ \\
\hline Presence of significant SAM & $75(50 \%)$ & $8(5.3 \%)^{*}$ \\
\hline Moderate or severe mitral regurgitation & $79(53 \%)$ & $4.2 \pm 0.7$ \\
\hline Left atrial size (cm) & $4.5 \pm 0.7$ & $34 \pm 10$ \\
\hline RVSP (mmHg) & $33 \pm 10$ & \\
\hline * P $<0.05$. LVOT, left ventricular outflow tract; SAM, systolic anterior motion; RVSP, right ventricular systolic pressure; Echo, echocardiogram.
\end{tabular}

mortality is $0.7 \%$ and there is clinically significant perioperative reduction in SAM and associated MR. In addition, there were no iatrogenic VSDs in our cohort and a low incidence of permanent pacemaker insertion. This confirms our previously published results, which have demonstrated excellent long-term survival of $98 \%$ at one year postoperatively, and extends the literature of contemporary surgical cohorts $(10,11)$.

As a dedicated centre for HOCM, we have seen a small evolution in referrals for patients. In particular, there have been increasing referrals for patients who are older with mild asymmetric hypertrophy who have refractory symptoms. These patients typically have a basal septal thickness $\leq 1.7 \mathrm{~cm}$ and include those with sigmoid septum anatomy or those with high dynamic gradients in the absence of obvious abnormalities in the sub-valvular apparatus. Despite the thin basal septal thickness in this small cohort, resolution of symptoms, perioperative mortality, insertion of pacemakers, and incidence of iatrogenic VSDs were not different between these subgroups. This finding is consistent with the consensus guidelines that advocate septal myectomy at dedicated centres for drug-refractory symptomatic HOCM patients, regardless of absolute basal septal thickness.

Recent use of three-dimensional echocardiography and magnetic resonance imaging (MRI) has identified HOCM patients with subtle findings of elongated mitral valves and displaced papillary muscles (12-17); however, guidelines as to when to intervene on the mitral-subvalvular apparatus in conjunction with LVOT obstruction in the setting of HOCM remains unclear and largely dictated by individual center practices. Some centres advocate performing additional mitral procedures in patients with a basal septum less than $1.8 \mathrm{~cm}$ (4). In our series, we demonstrate that in the absence of preoperative identified intrinsic mitral valve pathology, adequate septal myectomy is sufficient to abolish SAM and associated hemodynamically significant MR. This is in agreement with a recent study published by the Mayo Clinic $(18,19)$, which also showed that SAMrelated MR in obstructive HCM was effectively treated with adequate septal myectomy, with only $1.7 \%$ having residual $\geq$ grade $3 \mathrm{MR}$.

\section{Limitations}

This retrospective study describes isolated myectomy surgical outcomes at a single institution and is limited to reporting outcomes related to perioperative complications and echocardiographic findings associated with HOCM.

\section{Conclusions}

Our cohort demonstrates that isolated septal myectomy remains a safe and effective operation that can achieve excellent results, irrespective of basal septal thickness, when performed by experienced surgeons in a dedicated HCM center.

\section{Acknowledgements}

None.

\section{Footnote}

Conflicts of Interest: The authors have no conflicts of interest to declare. 


\section{References}

1. Gersh BJ, Maron BJ, Bonow RO, et al. 2011 ACCF/ AHA guideline for the diagnosis and treatment of hypertrophic cardiomyopathy: a report of the American College of Cardiology Foundation/American Heart Association Task Force on Practice Guidelines. Circulation 2011;124:e783-831.

2. Nagueh SF, Groves BM, Schwartz L, et al. Alcohol septal ablation for the treatment of hypertrophic obstructive cardiomyopathy. A multicenter North American registry. J Am Coll Cardiol 2011;58:2322-8.

3. Patel P, Dhillon A, Popovic ZB, et al. Left Ventricular Outflow Tract Obstruction in Hypertrophic Cardiomyopathy Patients Without Severe Septal Hypertrophy: Implications of Mitral Valve and Papillary Muscle Abnormalities Assessed Using Cardiac Magnetic Resonance and Echocardiography. Circ Cardiovasc Imaging 2015;8:e003132.

4. Thomas F, Rader F, Siegel RJ. The use of MitraClip for symptomatic patients with hypertrophic obstructive cardiomyopathy. Cardiology 2017;137:58-61.

5. Maron BJ, Dearani JA, Ommen SR, et al. Low operative mortality achieved with surgical septal myectomy: role of dedicated hypertrophic cardiomyopathy centers in the management of dynamic subaortic obstruction. J Am Coll Cardiol 2015;66:1307-8.

6. Woo A, Williams WG, Choi R, et al. Clinical and echocardiographic determinants of long-term survival after surgical myectomy in obstructive hypertrophic cardiomyopathy. Circulation 2005;111:2033-41.

7. Parry DJ, Raskin RE, Poynter JA, et al. Short and medium term outcomes of surgery for patients with hypertrophic obstructive cardiomyopathy. Ann Thorac Surg 2015;99:1213-9.

8. Ommen SR, Maron BJ, Olivotto I, et al. Long-term effects of surgical septal myectomy on survival in patients with obstructive hypertrophic cardiomyopathy. J Am Coll Cardiol 2005;46:470-6.

9. Ralph-Edwards A, Woo A, McCrindle BW, et al. Hypertrophic obstructive cardiomyopathy: comparison of outcomes after myectomy or alcohol ablation adjusted by propensity score. J Thorac Cardiovasc Surg 2005;129:351-8.

10. Maron BJ, Nishimura RA. Surgical septal myectomy versus alcohol septal ablation assessing the status of the controversy in 2014. Circulation 2014;130:1617-24.
11. Sorajja P, Valeti U, Nishimura RA, et al. Outcome of alcohol septal ablation for obstructive hypertrophic cardiomyopathy. Circulation 2008;118:131-9.

12. Kim DH, Handschumacher MD, Levine RA, et al. In vivo measurement of mitral leaflet surface area and subvalvular geometry in patients with asymmetrical septal hypertrophy: insights into the mechanism of outflow tract obstruction. Circulation 2010;122:1298-307.

13. Kaple RK, Murphy RT, DiPaola LM, et al. Mitral valve abnormalities in hypertrophic cardiomyopathy: echocardiographic features and surgical outcomes. Ann Thorac Surg 2008;85:1527-35, 1535.e1-2.

14. Harrigan CJ, Appelbaum E, Maron BJ, et al. Abnormal papillary muscle morphology is independently associated with increased left ventricular outflow tract obstruction in hypertrophic cardiomyopathy. Heart 2008;94:1295-301

15. Harrigan CJ, Appelbaum E, Maron BJ, et al. Significance of papillary muscle abnormalities identified by cardiovascular magnetic resonance in hypertrophic cardiomyopathy. Am J Cardiol 2008;101:668-73.

16. Maron MS, Olivotto I, Harrigan C, et al. Mitral valve abnormalities identified by cardiovascular magnetic resonance represent a primary phenotypic expression of hypertrophic cardiomyopathy. Circulation.2011;124:40-7.

17. Kwon DH, Smedira NG, Thamilarasan M, et al. Characteristics and surgical outcomes of symptomatic patients with hypertrophic cardiomyopathy with abnormal papillary muscle morphology undergoing papillary muscle reorientation. J Thorac Cardiovasc Surg 2010;140:317-24.

18. Hong JH, Schaff HV, Nishimura RA, et al. Mitral regurgitation in patients with hypertrophic obstructive cardiomyopathy implications for concomitant valve procedures. J Am Coll Cardiol 2016;68:1497-504.

19. Cho YH, Quintana E, Schaff HV, et al. Residual and recurrent gradients after septal myectomy for hypertrophic cardiomyopathy-mechanisms of obstruction and outcomes of reoperation. J Thorac Cardiovasc Surg 2014;148:90915; discussion 915-6.

Cite this article as: Vanderlaan RD, Woo A, Ralph-Edwards A. Isolated septal myectomy for hypertrophic obstructive cardiomyopathy: an update on the Toronto General Hospital experience. Ann Cardiothorac Surg 2017;6(4):364-368. doi: 10.21037/acs.2017.05.12 